\title{
Research on Key Problems of IPv6 Network Management System
}

\author{
Ping Tang ${ }^{1,}$, , Fuyu Tao ${ }^{1}$, Duo $\mathrm{Pei}^{2}$, Tao $\operatorname{Tan}^{3}$ \\ ${ }^{1}$ Chongqing Police College Chongqing China \\ ${ }^{2}$ Chongqing Economic and Information Commission Chongqing China \\ ${ }^{3} \mathrm{H} 3 \mathrm{C}$ Communication Technology Co, Ltd. \\ anotnull@163.com
}

Keywords: SNMP; neighbor discovery; Address Auto Configuration ; Information Acquisition.

Abstract. The new features of IPv6 lead to the lack of the existing network management system to support IPv6. The article focuses on the IPv6 neighbor discovery protocol, address auto configuration, and some the not accessible information in IPv6 MIB. The article also tries to solve the problems.

\section{Introduction}

IPv4 is the fourth version of the Internet protocol. It is also widely used and composition of the Internet technology today. But with the expansion of the scale of the network, the IPv4 has become a bottleneck of the development of the Internet. Limited address space, the rapid expanding of route form ,the lack of network security and imperfect QoS etc. have become the factors restricting the further development of the Internet. In order to thoroughly solve the problems about IPv4, the IETF has already begun to study the next generation Internet Protocol, IPv6. The IPv6 improved address space and application of network etc. to make it more suitable for current network development.

The progress of network technology drives the research of network management. Nowadays, both IPv6 and IPv4 are used. Therefore we need a network management system which can simultaneously monitor the IPv4 network and IPv6 network. However, because of the differences of two kinds of protocol, the network management system based on IPv4 can't fully competently manage the IPv6 network. Along with the development of the network management of the IPv6, we find that studying network management of the IPv6 based on the IPv4 network management combining with the IPv6 characteristics has become the priority of a new generation of network research.

\section{Introduction of the IPV6}

IPv6 is developed from the IPv4. So the IPv6 retains some advantage of the IPv4. At the same time, IPv6 also improved many defects of the IPv4. In general, the IPv6 has the following new features.[1]

I A larger address space

The IPv6 is mainly designed to solve the problem of shortage of IPv4 address .The address length of the IPv4 is 32 bits. That is to say the IPv4 has 232 addresses to assign. However, the address length of the IPv6 is 128 bits. 2128 addresses can give an IP address to every sand in the world. Estimate actual allocation of IPv6 address according to conservative approach, the whole earth can be assigned more than 1,000 addresses per square meter area.

I Simplified packet header

In order to reduce those who is not often used but still occupy statically space, IPv6 simplified packet header correspondingly. It speeds up the speed of the router to process the packet and improves the throughput of network as well.

I Better scalability and security

IPv6 adds extension header after IPv6 header. It makes IPv6 can expand the functions easily. Authentication header (AH) and encapsulation security payload (ESP) provide security controls of network layer. The AH used to ensure the consistency of the data and the ESP is used to ensure the confidentiality of data.

I Better Qos 
IPV6 defines the "traffic type" and "flow label" in the header. Intermediate routers can forward data belonging to the same stream intelligently. So it provides the better Qos and also fills the IPV4 defects.

I Address Auto Configuration

The IPV6 supports many address auto configurations, such as stateful address auto configuration and stateless address auto configuration. The stateful address auto configuration transformed from dynamic host configuration protocol (DHCP). The IPv6 inherited and improved the address auto configuration of the IPv4. In the stateless address auto configuration, the host generates a global unicast address by the RA message sent by the router on the receiving link.

\section{The Network Management}

Network management is a general name of supervision, organization, control and other activities which are required to meet network communication services and process information. The ISO in the document of ISO/IEC 7498-4 defines the basic functions of network management domain. We can know from the document of ISO/IEC 7498-4 that the basic function of network management includes fault management, configuration management, performance management, security management and flow management.[2]

Simple network management protocol (SNMP) was set by the IETF. Because it is easy to carry out, SNMP is one kind of successful network management protocol, which be used mostly in IP network. Along with the development of the Internet, from SNMPv1 to SNMPv2, to SNMPv3, the function of SNMP has been enriched and improved continuously. SNMP include structure of management information (SMI), management information base(MIB), protocol operations and definition formats of protocol data unit(PDU). [3]

The MIB determines the function that the network management can realize. It is the database that contains the information of the equipment resource and element. The managed device agent gets relevant information by accessing the MIB. The SMI defines and builds the data type of data which is used by managed objects in the MIB. And it defines the rules for describing management information, using ASN. 1 as well. That is to say, it provides a set of naming and defining rules for management objects. SNMP provides 5 PDUs. It is used for exchanging information between management processes and agents. These 5 PDUs are shown as follows. At the same time, These 5 PDUs are as shown in Figure 1.

I Get request: Extract one or more parameter values from the agent process;

I Get Next Request: Extract parameter values from agent process which is next to the current process.

I Set Request: Set one or more parameter values for the agent process.

I Get Response: Returns one or more parameter values. This operation is emitted by the agent process, which is the response of the previous three operations.

I Trap: Agent process to send the message, the management process to inform the management of certain things happen.[4]

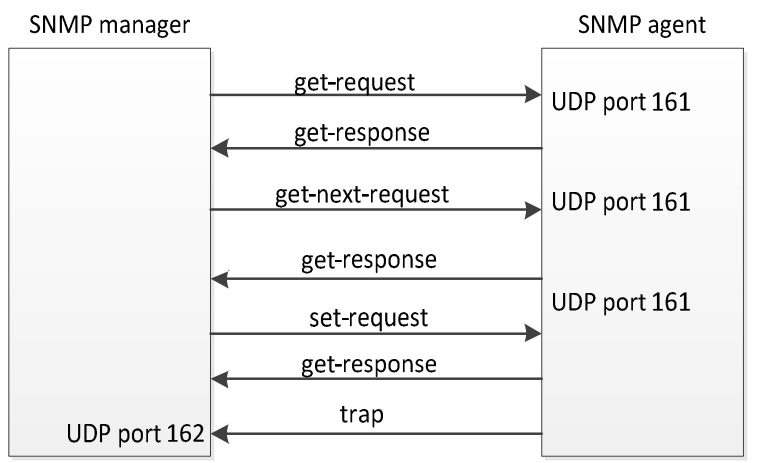

Figure 15 kinds of message operation of SNMP. 


\section{Key Problems of the IPV6 Network Management System}

As mentioned above, the IPv6 introduced many new features. At the same time the new features bring many new problems to network management. Then we will make a discussion on some of the key issues.

1) IPv6 neighbor discovery protocol

In IPV4 environment, when a network is built, at first the router the discover topology in its network. Then it records its knowledge of the network environment in the ARP table. It storages adjacent node`s IP address and other network routing information from. The SNMP manager under IPV4 environment can get the node`s information by reading the ARP table on the agent device. What's more, it can get the whole information of network topology by reading the ARP table on core router.

The IPV6 uses the IPv6 neighbor discovery protocol to replace the function of the ARP table and uses the extended ICMP protocol to carry the IPV6 packets. The IPV6 uses multicast to replace broadcast which is used by the IPV4 environment. It also defines several types of multicast address. In IPV6, the broadcast and the ARP table were canceled entirely. In IPV4, network management software by reading the router interface`s ARP table to get information subnet connected to the interface. The IPV6 also can use this method to obtain subnet information. However, it reads device ipv6NetToMediaTable in node`s MIB. The table is defined in RFC2465. Its oid value is 1.3.6.1.2.1.55.1.12. Network management software of manager can get the whole by the operation of GetNextRequest or GetRequest.[5]

The definition of part of ipv6NetToMediaTable is shown as follows.

ipv6NetToMediaEntry: : = SEQUENCE\{

ipv6NetToMediaNetAddress Ipv6Address,

ipv6NetToMediaPhysAddress physAddress,

ipv6NetToMediaType INTEGER,

ipv6NetToMediaState INTEGER,

ipv6NetToMediaLastUpdated TimeStamp,

\}

ipv6NetToMediaValid TruthValue

ipv6NetToMediaType OBJECT-TYPE

SYNTAX INTEGER \{

other(1),

dynamic(2),

static(3),

local(4),

\}

ipv6NetToMediaState OBJECT-TYPE

SYNTAX INTEGER \{

Reachable(1),

Stale(2),

Delay(3),

Probe(4),

Invalid(5),

Unknown(6),

\}

2) Address Auto Configuration:

The IPV6 has address auto configuration, which is not available in IPV4. How to manage the host which is used for dynamically assigning address is a key problem of network management. The best way is to use the domain name. If the server provide services to the client, it will use the domain name instead of the IP address. 
In IPV6 environment, the hosts address changes as the change of access points and routers advertisement prefix. This automatic configuration put forward a very high request of the DNS server. It requires the server to update in a timely manner and to establish an effective binding of address and domain name. The management system must use the domain name and IP address to identify the host. It can use the domain name or IP address to identify the host which has the fixed address. But the host which has dynamic address, a domain name should be managed by the domain name.

The ability of the DNS server automatic updating is shown as follows. At first, the server assigns a name for each static host. When the host dynamically get IPV6 address, it will notify the domain name server in time and establish a link between name and address. Then it will inform the change to managers. The network management system should also be adjusted accordingly.

3) Information Acquisition

Information acquisition is the most important part of the network management. All of fault management, configuration management, performance management, security management and flow management need to collect routers, servers and other data information in the framework of the SNMP from managed equipment. Precisely, it acquires the data in the MIB. Nowadays, the IETF has put forward some definition about IPv6 MIB. However some network equipment is not support for the IPv6 MIB. It make traditional network management technology based on SNMP cannot access network management information. Some network management information is not accessible. It caused the barriers to get information from the IPv6 equipment. So we cannot use the SNMP protocol to obtain related network management information from the network equipment directly. In order to solve the problem, people have put forward using mobile agent or proxy agent or Telnet/SSh to get the information in the MIB.

Mobile agent (MA) requires the managers and agents to equip with mobile agent server. If we need to collect the not accessible data in the IPv6 MIB, the manager will generate a MA to be sent to the managed equipment to directly read data in a local document. So it needs not to deal with the SNMP agent[7]. Structure of network management using mobile agent is shown as Figure 2.

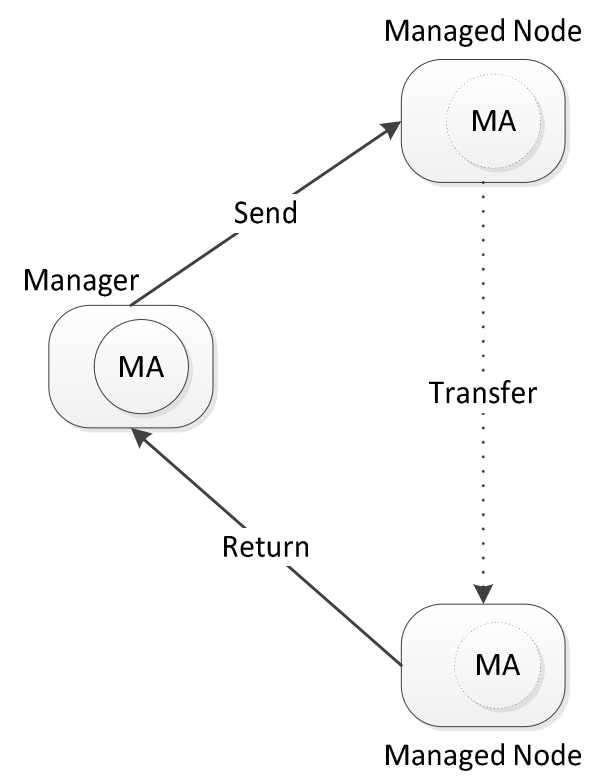

Figure 2 Structure of network management using mobile agent

Another solution to solve the "IPV6 not accessible" is the Telnet/SSh. When managers need not read the not accessible data in IPV6 MIB, it can establish a secure Telnet login by Telnet/SSH way, and then access to data. The above process can be abstracted as a proxy agent. It likes adding an agent between managers and agents to obtain the not accessible data in IPV6 MIB. The workflow of Telnet/SSH is shown as Figure 3. 


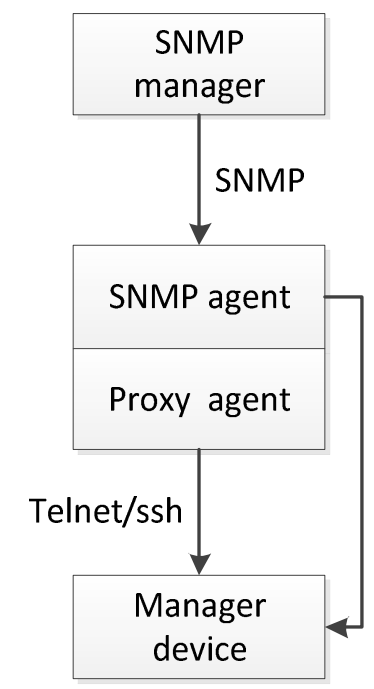

Figure 3 Workflow of Telnet/SSH

\section{Summary}

Network management technology has become more and more mature with the development of the IPv4. However, the IPv6 network brings some new network management problems. It makes the traditional network management technology meet the bottlenecks.

The paper analyzes the change of the network management technology brought by the new characteristics of the IPv6. The paper also tried to put forward the corresponding solutions. It is very meaningful to study out the mature IPv6 network management system as soon as possible.

\section{Acknowledgements}

This work was financially supported by the research project KJ1501508 from Chongqing Education Committee.

\section{References}

[1] LIU Ying, REN Xin-hua, DUAN Lin-mao, PAN Li-hu, "The Discussion of SNMP Transition Mechanism From IPv4 to IPv6," JOURNAL OF TAI YUAN UNIVERSITY OF TECHNOLOGY, Vol.35 NO.6, Taiyuan ,Nov 2004.

[2] LU Wei, HUANG Rui, YAN Bin-yu, "Design and Application of a Simple Network Management System Based on SNMP," Research of application of computer, Vol.35 NO.6, Chendu Sichuan, 2002.

[3] CAI Li, ZHANG Da-fang,XIE Gao-gang, "Design and Implementation of Network Management System Based on SNMP,” Computer Applications, Vol.23 NO.3, Changsha Hunan, Mar. 2003

[4] CUI Ran HUA Gang, "Design of network management system based on SNMP," Network and Comunication, Vol.25 NO.6-3, Xuzhou Jiangsu, 2009.

[5] TANG Xue-wen , XIONG Zhong-yang, WANG Bao, LIU Ye-xin, "Campus network IPv6 network management system design and implementation," Journal of Guangxi University: Nat Sci Ed, Vol.36 Sup 1, Chongqing, 2011.

[6] LU Cheng-mao, XIONG Qi-bang, "A New Solution for IPv6 Transition Network Management," Computer Technology and Development, Vol.16, No 1, Shang hai, Jan 2006.

[7] Tan Xingli, "Research and Implementation of Integrated Network Management Supports IPv6," College of Computer Science of Chongqing University Chongqing. April, 2013. 